\title{
Random walks with intersections: Static and dynamic fractal properties
}

\author{
Raf Dekeyser \\ Instituut voor Theoretische Fysica, Katholieke Universiteit Leuven, B-3030 Leuven, Belgium \\ Amos Maritan* and Attilio Stella ${ }^{\dagger}$ \\ Dipartimento di Fisica "Galileo Galilei" dell'Università degli Studi di Padova, I-35131 Padova, Italy \\ (Received 20 April 1987)
}

\begin{abstract}
Static and dynamic properties of the fractal sets generated by free and $k$-tolerant walks are analyzed in detail. A rather complete picture is obtained for the set of the intersections of free random walks, using correlation functions like the probability of visiting one or more sites $m$ times. Monte Carlo enumerations, jointly with rather sophisticated numerical analysis, are used to determine the fractal dimension of the set of the self-intersections of $k$-tolerant walks. The results are used to throw new light on the Flory argument for polymer chains with excluded-volume effects; the universal behavior of $k$-tolerant walks is explained in a coarse-grained reinterpretation of the Flory approximation. Diffusion on the same class of walks allows us to discuss also their universality with respect to dynamical properties. In particular, a spectral dimension equal to $\frac{4}{3}$ is obtained for the free random walk in $d=2$ dimensions.
\end{abstract}

\section{INTRODUCTION}

In spite of their relevance for many problems of polymer physics and lattice statistics, intersection properties of random fractals did receive relatively little attention up to now in the physical literature.

These properties are obviously connected with many important issues, like the upper critical dimension of geometric or magnetic problems, or the well-known Flory approximation of polymer statistics. ${ }^{1,2}$ The upper critical dimension of a model is known to depend crucially on the properties of mutual intersections of two replicas of the fractal under consideration. In the Flory approach, on the other hand, one tries to estimate the repulsive energy due to excluded-volume effects in a polymer (e.g., linear, or branched, etc.) as being proportional to the number of self-intersections of a free, unconstrained realization of the chain.

This paper presents a systematic investigation of mutual and self-intersection properties of random fractals on a lattice. The purpose of this investigation is first of all to clarify and base on a firm ground the fundamental laws governing the fractal geometry of intersections of fractals. To this purpose we present an extended set of analytical and numerical results concerning, respectively, free random walks and $k$-tolerant walks on the lattice. The discussion of free random walks is mostly based on wellestablished results; ${ }^{3}$ what is to some extent original is the way in which these results are used to highlight basic facts about fractal properties of intersections, which were somehow disregarded or misinterpreted by previous authors.

This part of the work also contains a calculation of multiple-point correlation functions for the free random walk (see also the Appendix), which, to our knowledge, was never produced before.
The $k$-tolerant walks are taken as examples of nontrivial fractals allowing for self-intersections. For these walks, our analysis has been based on numerical methods; in particular, to test the fractal properties of selfintersections, we had to analyze Monte Carlo enumeration results with rather sophisticated extrapolation techniques, e.g., based on Padé approximants.

The main result of this first (static) part of this paper is the nontrivial reinterpretation of the Flory approximation for polymer statistics, given in Sec. V. Apart from showing the deep connection existing between Flory's result and a correct application of the fractal geometry of intersections, this development has the very important merit of clarifying for the first time the scaling-invariance properties behind the construction of the Flory free energy, and the degree of universality which can be expected from the predictions of the approach. As an example, we give for the first time a correct Flory prediction for $k$-tolerant walks, thus succeeding where previous attempts, based on standard derivations, failed. ${ }^{4}$

If the role of fractal-intersection properties of random sets in static problems, like $k$-tolerant walks, can to some extent be understood (and this paper hopefully brings a substantial contribution to such understanding), the effect of these properties on the dynamical problems on fractal structures is much less clear. This is a subject of considerable present interest, in connection with many important physical issues, ranging from transport in disordered structures, to low-frequency vibrational spectra of biomolecules. 5,6

A question which can, e.g., be raised in this context, is whether, when considering a $k$-tolerant walk, this model should possess the same spectral dimension as a simple nonintersecting self-avoiding walk (SAW). This dimension $\widetilde{d}$, which is related to the properties of diffusion on the structure, or to the density of vibrational modes, is 
simply equal to 1 for a topologically linear structure (SAW). One could suspect that, while the loop structure compatible with the $k$-tolerance is not able to modify the static fractal dimension of the SAW, it could have effects on the dynamical spectral dimension. Sections VI and VII present some original results of extensive simulations of diffusions on self-avoiding walks with bridges on $k$ tolerant walks and on free random walks in two dimensions. These results are of relevant pedagogical value, especially in connection with the problem of explaining anomalous temperature dependence of ESR relaxation times measured on proteins at low temperatures. ${ }^{7}$

The results of Sec. VII, on the spectral properties of the random walk in two dimensions, give, to our knowledge, the first sharp and convincing numerical and theoretical prediction concerning a very controversial and rather obscure issue. This subject is, in our opinion, a most challenging one, on which one should test our fundamental understanding of the whole subject of fractal dynamics. Free walks on the lattice are perhaps the most elementary conceivable random fractals. On the other hand, their dynamical properties were still obscure in $d=2.8,9$ The presence of logarithmic corrections in the asymptotic laws, which we established, give somehow an a posteriori justification for the difficult and controversial character of this subject.

The paper is organized as follows. Section II describes some basic definitions and properties of mutual and selfintersection sets, which are then illustrated analytically in Sec. III on free random walks and investigated numerically in Sec. IV in the case of $k$-tolerant walks. In Sec. V these properties are used as cornerstones for a new analysis of the Flory argument for the fractal dimension of geometrical problems in general and self-avoiding and $k$-tolerant walks in particular. Sec. VI is devoted to a numerical investigation of the dynamical universality of the same class of restricted walks, whereas Sec. VII describes the dynamical fractal behavior of free random walks in $d=2$.

\section{SCALING OF MUTUAL INTERSECTIONS AND SELF-INTERSECTIONS OF RANDOM WALKS}

In this section we consider an arbitrary class of random walks of unit steps on a regular $d$-dimensional lattice. This means that the class of walks may correspond to some prescription for an excluded-volume effect, or it may be the class of free random walks. We associate a fugacity $K$ to each step. The two-point Green function is defined in the standard way as

$$
G(\mathbf{x}, \mathbf{y})=\sum_{\substack{W \\ W=\{\mathbf{x}, \mathbf{y}\}}} K^{|W|}
$$

where $|W|$ is the number of steps in the walk $W$ and $\partial W$ is its boundary; $\mathbf{x}$ and $\mathbf{y}$ are arbitrary lattice sites. If $\mathbf{x}=\mathbf{y}$, the sum has to contain also the zero-step walk.

It seems reasonable to assume that there exists some characteristic length $\xi$, diverging as $\left(K_{c}-K\right)^{-v}$ when $K$ approaches the critical fugacity $K_{c}$ from below. This length has the property that

$$
\begin{array}{r}
G(\mathbf{x}, \mathbf{y}) \sim|\mathbf{x}-\mathbf{y}|^{2-d-\eta} F(|\mathbf{x}-\mathbf{y}| / \xi) \\
\text { as }|\mathbf{x}-\mathbf{y}| \rightarrow \infty,
\end{array}
$$

where $F(z)$ is an exponentially decreasing function at $z \rightarrow \infty$.

Another interesting correlation function ${ }^{10}$ is the grandcanonical probability for a walk to visit a given site $\mathbf{x}$, when it starts at the origin,

$$
P(\mathbf{x})=\sum_{W_{0}} K^{\left|W_{0}\right|} \chi_{W_{0}}(\mathbf{x})\left(\sum_{W_{0}} K^{\left|W_{0}\right|}\right)^{-1},
$$

where the characteristic function $\chi_{W}$ is 1 if $W$ visits $\mathbf{x}$, and zero otherwise. $W_{0}$ is a walk starting at the origin. Scaling requires that for $|\mathbf{x}| \rightarrow \infty$ and $K \rightarrow K_{c}^{-}$,

$$
P(\mathbf{x}) \simeq|\mathbf{x}|^{-\phi} f(|\mathbf{x}| / \xi)
$$

where the function $f(y)$ is expected to approach a constant value for $y \rightarrow 0$. The exponent $\phi$ is related to the fractal dimension of the walk. ${ }^{2}$ Indeed, one has

$$
\sum_{\mathbf{x}} P(\mathbf{x})=\left\langle\left\|W_{0}\right\|\right\rangle
$$

where $\|W\|$ is the number of distinct sites visited by a walk $W$.

A possible definition of the fractal dimension of the considered class of walks is through the relation

$$
\langle\|W\|\rangle \sim \xi^{D} \text { as } K \rightarrow K_{c}^{-}
$$

From (2.4), this implies that

$$
\phi=d-D
$$

On an intuitive basis, this means that $P(\mathbf{x})$ in $(2.4)$ determines the average density of sites visited by $W_{0}$.

The average number of steps in the walks in our grand-canonical ensemble behaves asymptotically (for $K \rightarrow K_{c}^{-}$) as

$$
\left\langle\left|W_{0}\right|\right\rangle=K \frac{\partial}{\partial K} \ln \sum_{\mathbf{x}} G(\mathbf{O}, \mathbf{x}) \sim \xi^{1 / v} \sim\left\langle\left\|W_{0}\right\|\right\rangle^{1 / D v}
$$

By the definitions of $|W|$ and $\|W\|$, we must have $D v \leq 1$. For free random walks in $d \geq 2$, it is known that $D v=1 .^{3}$ Excluded-volume effects will only reduce the number of multiple points, and we therefore expect this equality to hold a fortiori on models with repulsive interactions. We have only in $d=1$ that for free random walks $\left\langle\left|W_{0}\right|\right\rangle \sim \xi^{2}$ and $\left\langle\left\|W_{0}\right\|\right\rangle \sim \xi$, which gives $D=1$, $v=\frac{1}{2}$.

Let us now consider two independent walks, $W_{1}$ and $W_{2}$, from the same class with the same origin. We can calculate the average number of mutual intersection points as 


$$
\begin{aligned}
\left\langle\left\|W_{1} \cap W_{2}\right\|\right\rangle & =\sum_{W_{1}, W_{2}} K^{\left|W_{1}\right|+\left|W_{2}\right|} \| W_{1} \cap W_{2}||\left(\sum_{W_{1}, W_{2}} K^{\left|W_{1}\right|+\left|W_{2}\right|}\right]^{-1} \\
& =\sum_{W_{1}, W_{2}} K^{\left|W_{1}\right|+\mid W_{2}} \sum_{\mathbf{x}} \chi_{W_{1}}(\mathbf{x}) \chi_{W_{2}}(\mathbf{x})\left(\sum_{W_{1}, W_{2}} K^{\left|W_{1}\right|+\left|W_{2}\right|}\right]^{-1} \\
& =\sum_{\mathbf{x}}[P(\mathbf{x})]^{2} \sim \xi^{2 D-d} \text { as } K \rightarrow K_{c}^{-} .
\end{aligned}
$$

Equation (2.9) states that the fractal dimension of the mutual intersection points of the walks is

$$
D\left(W_{1} \cap W_{2}\right)=2 D-d,
$$

which is a particular case of the codimension additivity law. In its general form, ${ }^{11}$ this law states that the fractal dimension of the intersection set $A \cap B$ of two fractals $A$ and $B$, with respective fractal dimensions $D_{A}$ and $D_{B}$ in a $d$-dimensional space, is given by

$$
D(A \cap B)=\max \left\{D_{A}+D_{B}-d, 0\right\} .
$$

If, on the contrary, we ask for the fractal dimension of the set of self-intersection points of a single walk $W$ (assuming that this walk allows such intersections), the situation is less clear. In the literature ${ }^{4}$ it is often assumed that the fractal dimension of the set of sites that are visited $m$ times by $W$ ( $m$-multiple intersection points) is the same as the fractal dimension of $m$ independent replicas of $W$. Using Eq. (2.11), this means $\max [m D-(m-1) d, 0]$. This replica idea, however, is contradicted both by exact results for free random walks (see Sec. III) and by extensive numerical investigations of $k$-tolerant walks in $d=2$ and 3 (see Sec. IV).

To conclude this section let us generalize some of our previous definitions to the set of multiple points. If $\chi_{W_{0}}^{(m)}(\mathbf{x})$ is the characteristic function of the $m$-multiple points, we define

$$
P_{m}(\mathbf{x})=\sum_{W_{0}} K^{\left|W_{0}\right|} \chi_{W_{0}}^{(m)}(\mathbf{x})\left(\sum_{W_{0}} K^{\left|W_{0}\right|}\right)^{-1} .
$$

Again assuming scaling, now with a correlation length $\xi_{m}$, one can argue that

$$
P_{m}(\mathbf{x})=|\mathbf{x}|^{D_{m}-d} f\left(|\mathbf{x}| / \xi_{m}\right),
$$

where $D_{m}$ is the fractal dimension of the set of $m$-multiple points. The average number of such points is then

$$
\left\langle\|W\|_{m}\right\rangle=\sum_{\mathbf{x}} P_{m}(\mathbf{x}) \sim \xi_{m}^{D_{m}} \text { as } K \rightarrow K_{c}^{-} \text {. }
$$

In principle, $\xi_{m}$ may be different from the correlation length $\xi$, defined previously. In order to have a precise definition of it, we should also study a suitable generalization of (2.1), e.g., the function

$$
G_{m}(\mathbf{x}, \mathbf{y})=\sum_{W} \chi_{W}^{(m)}(\mathbf{x}) \chi_{W}^{(m)}(\mathbf{y}) K^{|W|}
$$

where $W$ is a generic random walk, with arbitrary starting point on the lattice.
We must remark that in (2.9) we implicitly assumed that the correlation length of $W_{1} \cap W_{2}$ was the same as that of the separate walks. It is obvious that our conclusions are insensitive to changes in the definition of $\xi$, if in the $K \rightarrow K_{c}^{-}$limit they reduce to a simple multiplicative factor. In practical calculations we therefore will often use other length scales proportional to $\xi$ as, e.g., the endto-end distance of a walk, or the mean radius of gyration of a set of points. In the next sections, we will come back to some of the properties for free and $k$-tolerant walks.

\section{EXACT RESULTS FOR FREE RANDOM WALKS}

It is quite instructive to illustrate the validity of some hypotheses, made in Sec. II, in the case of free random walks on a hypercubic lattice in $d$ dimensions, for which obviously

$$
\sum_{\mathbf{x}} G(0, \mathbf{x})=(1-2 K d)^{-1} .
$$

This means that $K_{c}=(2 d)^{-1}$, and from (2.8) we have

$$
\langle|W|\rangle=\frac{2 K d}{1-2 K d} \sim \frac{K_{c}}{K_{c}-K} \quad \text { as } K \rightarrow K_{c}^{--} .
$$

The Green function itself was derived by Montroll and Weiss, ${ }^{3}$ and is given by

$$
\begin{aligned}
G(\mathbf{x}, \mathbf{y})= & \int_{-\pi}^{\pi} \frac{d^{d} \mathbf{p}}{(2 \pi)^{d}} \frac{e^{i \mathbf{p} \cdot(\mathbf{x}-\mathbf{y})}}{1-2 K \sum_{j} \cos p_{j}} \\
& \sim|\mathbf{x}-\mathbf{y}|^{2-d}\left[\frac{\xi}{|\mathbf{x}-\mathbf{y}|}\right)^{(3-d) / 2} \\
& \times \exp \left(-\frac{|\mathbf{x}-\mathbf{y}|}{\xi}\right) \text { as } K \rightarrow K_{c}^{-} ;
\end{aligned}
$$

asymptotically $\xi$ behaves as $\sqrt{K_{c}-K}$, which means $v=\frac{1}{2}$.

In order to calculate the correlation function $P(\mathbf{x})$, it is sufficient to consider the related function

$G_{1}(\mathbf{x})=\sum_{\substack{W \\ \partial W=\{0, \mathbf{x}\}}} K^{|W|} \chi_{W}^{(1)}(\mathbf{x})=\frac{G(0, \mathbf{x})-\delta_{\mathbf{x}, 0}}{G(0,0)}$

(see again Ref. 3 ). $G_{1}(\mathbf{x})$ contains the sum over all random walks, whose final step visits $\mathbf{x}$ for the first time. $G_{1}(0)$ is the sum over the nontrivial walks returning for 
the first time to the origin, and

$$
G(0,0)=\sum_{n=0}^{\infty}\left[G_{1}(\mathbf{0})\right]^{n}
$$

Using the definition (2.3) it is easy to show that for $\mathbf{x} \neq 0$, the following holds:

$$
\begin{aligned}
P(\mathbf{x}) & =\sum_{\substack{W \\
\partial W=\{0, \mathbf{x}\}}} K^{|W|} \chi_{W}^{(1)}(\mathbf{x}) \sum_{W_{\mathbf{x}}} K^{\left|W_{\mathbf{x}}\right|}\left(\sum_{W_{0}} K^{\left|W_{0}\right|}\right)^{-1} \\
& =G_{1}(\mathbf{x})=\frac{G(\mathbf{0}, \mathbf{x})}{G(\mathbf{0}, \mathbf{0})}
\end{aligned}
$$

We denoted by $W_{\mathbf{x}}$ the class of walks that start at $\mathbf{x}$, and we used the fact that they are just a translation by $\mathbf{x}$ of the class $W_{0}$. Since $P(\mathbf{0})=1$, the final result also holds for $\mathbf{x}=\mathbf{0}$.

Montroll and Weiss proved furthermore that for $K \rightarrow K_{c}^{-}$,

$$
G(0,0) \rightarrow\left\{\begin{array}{l}
\text { const for } d>2 \\
\ln \xi \text { for } d=2 \\
\xi^{2-d} \text { for } d<2
\end{array}\right.
$$

from which we deduce that in the same limit and for $(|\mathbf{x}| / \xi) \rightarrow \infty$ we have

$$
P(\mathbf{x}) \rightarrow\left\{\begin{array}{l}
|\mathbf{x}|^{2-d} f_{d}(|\mathbf{x}| / \xi) \text { for } d>2 \\
f_{2}(|\mathbf{x}| / \xi)(\ln \xi)^{-1} \text { for } d=2 \\
f_{d}(|\mathbf{x}| / \xi) \text { for } d<2 .
\end{array}\right.
$$

This implies the well-known result ${ }^{12,13}$ that the fractal dimension is given by

$$
D=\min \{d, 2\} \text {. }
$$

Considering now the intersection of two independent free random walks (see also Ref. 14), (2.9) immediately leads to

$$
D\left(W_{1} \cap W_{2}\right)=\min \{d, 4-d\} \text { for } d \leq 4 .
$$

For $d>4$, the intersection set is finite and has no fractal properties.

It may be interesting to note that

$$
\begin{aligned}
\left\langle\| W_{1}\right. & \left.\cap W_{2} \|\right\rangle \\
& =\sum_{\mathbf{x}}[G(\mathbf{0}, \mathbf{x})]^{2}[G(\mathbf{0}, \mathbf{0})]^{-2} \\
& \left.\left.=\iint d^{d} \mathbf{p} \widetilde{G}(\mathbf{p}) \widetilde{G}(-\mathbf{p})\right] \iint d^{d} \mathbf{p} \widetilde{G}(\mathbf{p})\right]^{-2},
\end{aligned}
$$

where $\widetilde{G}(\mathbf{p})$ is the Fourier transform of $G(\mathbf{x}, \mathbf{y})$ [see Eq. (3.3)]. (3.11) can simply be interpreted as the first-order perturbation correction to the one-particle irreducible vertex function at zero external wave vectors of a $\phi^{4}$-field theory in its regular lattice representation. The link between scalar field theory and random walks is not new. ${ }^{15}$ Up to our knowledge, however, this link was never exploited for studying the intersection points of independent walks. Since (3.11) diverges at $K_{c}$ for $d \leq 4$ and is finite for $d>4$, one sees that the infrared behavior of a $\phi^{4}$ theory is directly related to the fractal properties of the intersections of independent random walks.

Next, we consider the set of $m$-multiple intersection points of a free random walk. Montroll and Weiss ${ }^{3}$ showed how one can calculate the average number of points visited exactly $m$ times in such walks with a given number $|W|$ of steps. Indeed, with a simple generalization of the argument leading to (3.6), one gets

$P_{m}(\mathbf{x})=G(\mathbf{0}, \mathbf{x})[G(0,0)-1]^{m-1}[G(0,0)]^{-m-1}$.

Details of the derivation of this and subsequent formulas are given in the Appendix. Summing (3.12) over $\mathbf{x}$ and using the previous asymptotic expressions for $G(0,0)$ and $G(\mathbf{0}, \mathbf{x})$, one obtains that for $K \rightarrow K_{c}^{-}$,

$$
\left\langle\|W\|_{m}\right\rangle \rightarrow\left\{\begin{array}{l}
\xi^{2} \text { for } d>2 \\
\xi^{2}(\ln \xi)^{-2} \text { for } d=2 \\
\xi^{2 d-2} \text { for } d<2 .
\end{array}\right.
$$

This implies that, asymptotically for $\langle|W|\rangle \rightarrow \infty$,

$$
\left\langle\|W\|_{m}\right\rangle \rightarrow\left\{\begin{array}{l}
\langle|W|\rangle \text { for } d>2 \\
\langle|W|\rangle(\ln \langle|W|\rangle)^{-2} \text { for } d=2 \\
\langle|W|\rangle^{d-1} \text { for } d<2 .
\end{array}\right.
$$

In $d=1$, the number $\left\langle\|W\|_{m}\right\rangle$ is constant (no logarithmic correction).

For $d>2$ we can also determine the ratio between $\left\langle\|\boldsymbol{W}\|_{m}\right\rangle$ and $\langle|W|\rangle$ as a function of $m$. Indeed, from (3.1), (3.2), (3.12), and (2.14) it follows that

$$
\begin{aligned}
& \frac{\left\langle\|W\|_{m}\right\rangle}{\langle|W|\rangle} \simeq[G(\mathbf{0}, \mathbf{0})]^{-2}\left\{1-[G(\mathbf{0}, \mathbf{0})]^{-1}\right\}^{m-1} \\
& \text { as } K \rightarrow K_{c}^{-} \text {. }
\end{aligned}
$$

Summing (3.12) also over $m$, we obtain the average number of distinct visited sites as

$$
\langle\|W\|\rangle=\frac{K_{c}}{K}\langle|W|\rangle[G(\mathbf{0 , 0})]^{-1},
$$

from which we see that asymptotically for $K \rightarrow K_{c}^{-}$(or $\langle|W|\rangle \rightarrow \infty)$

$$
\langle\|W\|\rangle \sim\left\{\begin{array}{l}
\langle|W|\rangle \text { for } d>2 \\
\langle|W|\rangle[\ln \langle|W|\rangle]^{-1} \text { for } d=2 \\
\langle|W|\rangle^{d / 2} \text { for } d<2 .
\end{array}\right.
$$

Before drawing any conclusions from (3.13) concerning the fractal dimensions of these intersection sets, we should first investigate the correlation function $G_{m}(\mathbf{x}, \mathbf{y})$, in order to determine the relation between $\xi_{m}$ and $\xi$. It is rather simple to obtain, in a heuristic way, the asymptotic behavior of $G_{m}(\mathbf{x}, \mathbf{y})$ for the free walk in the $|\mathbf{x}-\mathbf{y}| \rightarrow \infty$ limit. Let us suppose that the walk starts at a given site $\mathbf{u}$ and reaches a final site $\mathbf{v}$, visiting both $\mathbf{x}$ and $\mathbf{y} m$ times in between. When the distance between $\mathbf{x}$ and $\mathbf{y}$ is very large, 
the probability that such a walk crosses this distance more than once is very small; such walks that travel up and down more than once indeed give a contribution of order $G(\mathbf{x}, \mathbf{y})^{n}$ with $n>1$. The main contribution derives from walks that can be divided in three parts: (a) a walk starting at $\mathbf{u}$ and reaching $\mathbf{x}$ for the $m$ th time at the last step, (b) a walk leaving $\mathbf{x}$ without coming back to it, and reaching $\mathbf{y}$ for the first time at the final step, (c) a walk starting at $\mathbf{y}$ and reaching $\mathbf{v}$ after coming back $(m-1)$ times to $\mathbf{y}$. Taking into account also the walks where the role of $\mathbf{x}$ and $\mathbf{y}$ is interchanged, and summing over $\mathbf{u}$ and $\mathbf{v}$, we obtain

$$
\begin{aligned}
G_{m}(\mathbf{x}, \mathbf{y}) & \sim 2 \sum_{\mathbf{u}, \mathbf{v}} \frac{G(\mathbf{u}, \mathbf{x})}{G(\mathbf{x}, \mathbf{x})}\left[\frac{G(\mathbf{x}, \mathbf{x})-1}{G(\mathbf{x}, \mathbf{x})}\right]^{m-1} \frac{G(\mathbf{x}, \mathbf{y})}{G(0,0)^{2}}\left[\frac{G(\mathbf{y}, \mathbf{y})-1}{G(\mathbf{y}, \mathbf{y})}\right)^{m-1} \frac{G(\mathbf{y}, \mathbf{v})}{G(\mathbf{y}, \mathbf{y})} \text { as }|\mathbf{x}-\mathbf{y}| \rightarrow \infty \\
& =2 G(\mathbf{x}, \mathbf{y})\left(\sum_{\mathbf{z}} G(0, \mathbf{z})\right]^{2}[G(0,0)-1]^{2 m-2}[G(0,0)]^{-(2 m+2)}
\end{aligned}
$$

This demonstrates that, apart from a multiplicative constant, $G_{m}$ behaves asymptotically as $G$, from which follows that $\xi_{m}=\xi$. There is only one correlation length for the free random walk. A more rigorous procedure for deriving Eq. (3.18) is outlined in the Appendix.

From (2.14) and (3.13) it then follows that the fractal dimension of the $m$-multiple self-intersections is

$$
D_{m}=\min \{2,2 d-2\} \text { for } d \geq 1,
$$

apart from a logarithmic correction in $d=2$. Comparing this with (3.9), we remark that $D_{m}$ is equal to $D$ for $d \geq 2$, but $D_{m}<D$ for $1 \leq d<2$. However, $D_{m}$ is never equal to the fractal dimension of the intersection set of $m$ independent replicas, except for $d=2$. In Fig. 1 we graphically combine various results obtained in this section for the fractal dimensionalities of different sets. We see no reason why a repulsive interaction could possibly change the fact that mutual intersection sets have a different fractal dimension from the self-intersection sets.

\section{NUMERICAL STUDIES OF $k$-TOLERANT WALKS}

In order to confirm some of the statements and assumptions made in Secs. II and III, we performed several numerical studies on the $k$-tolerant walks.

First, we have generated 2-tolerant walks with up to 50

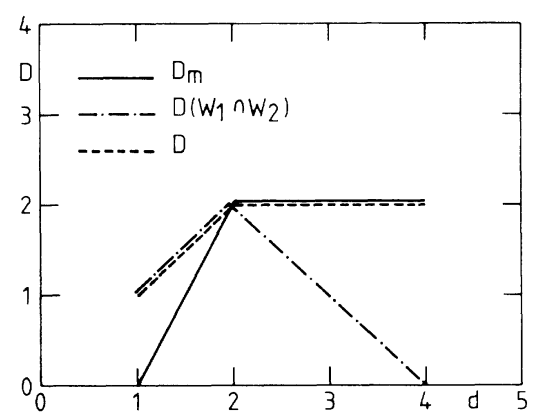

FIG. 1. Fractal dimensions $D(\ldots--)$ of free random walks, $D\left(W_{1} \cap W_{2}\right)(-\cdot-\cdot-\cdot)$ of the intersection set of two such independent walks, and $D_{m}(-)$ of the set of $m$ multiple self-intersection points, as functions of the Euclidean dimension $d$. steps, both on the triangular and on the cubic lattice. Our sample consisted of $1.6 \times 10^{6}$ (triangular), respectively $2.10^{6}$ (simple cubic) walks, and they were given relative weights according to the method described by Rosenbluth and Rosenbluth ${ }^{16}$ for the self-avoiding walks. A preliminary calculation was based on a $4 \times 10^{5}$ sample of walks with up to 45 steps on the square lattice. For each number of steps, up to the maximum, we calculated the average end-to-end square distance, and the average number of double points. We have developed special methods of series analysis, suitable for treating series generated by Monte Carlo methods. Using these techniques, which will be described in detail in a separate paper, we first of all confirmed that these 2-tolerant walks belong to the SAW universality class, since the end-to-end square distance after $n$ steps behaves as

$$
\left\langle R_{n}^{2}\right\rangle \sim n^{2 v}
$$

where $v=0.75 \pm 0.01$ in $d=2$ and $v=0.58 \pm 0.01$ in $d=3$, corresponding to fractal dimensionality $D=\frac{4}{3}$ and 1.72 , respectively. This point, however, was already made clear in previous publications. ${ }^{10,17}$

Next, we have analyzed the number of double points on these walks, in order to verify whether this number is proportional to the length of the walk, as in the free random walk case. We obtained indeed for the 2-tolerant walks that

$$
f_{2}(n) \equiv\left\langle\left\|W_{2}\right\|\right\rangle_{(|W|=n)} \rightarrow n a,
$$

where

$$
\begin{aligned}
& (a)_{\text {square }}=0.226 \pm 0.001, \\
& (a)_{\text {triangular }}=0.214 \pm 0.001, \\
& (a)_{\text {simple cubic }}=0.168 \pm 0.001 .
\end{aligned}
$$

In contrast to the free random walk case (3.11), however, there is no logarithmic correction in $d=2$. This can immediately be seen from Table I, where we collected for some values of $n$ the corresponding values of $f_{2}(n) / n$ and $f_{2}(n)\left(\ln ^{2} n\right) / n$ for the triangular lattice. Only the first numbers seem to tend to a constant value.

We have also generated some 5-tolerant walks on the square lattice. It is obvious, though, that here one has to 
TABLE I. Some values of $f_{2}(n) n^{-1}$ and $f_{2}(n) n^{-1} \ln ^{2} n$, where $f_{2}(n)=\left\langle\|W\|_{2}\right\rangle_{(|\boldsymbol{W}|=n)}$ for 2-tolerant walks on the triangular lattice. The first column extropolates to 0.214 .

\begin{tabular}{lcc}
\hline$n$ & $f_{2}(n) n^{-1}$ & $f_{2}(n) n^{-1} \ln ^{2} n$ \\
\hline 10 & 0.18954 & 1.0049 \\
20 & 0.20258 & 1.8181 \\
30 & 0.20672 & 2.3914 \\
40 & 0.20876 & 2.8408 \\
50 & 0.20984 & 3.2113 \\
\hline \hline
\end{tabular}

go to much higher lengths of walks, in order to detect the SAW nature of these walks: short 5-tolerant walks behave almost like free random walks. Therefore, the techniques of series analysis up to 50 terms seemed not appropriate. We generated samples of $10^{4}$ walks for each of the lengths $n=10,20,30,50,200,300$, and 500. Using the formula

$$
v \simeq \frac{1}{2} \frac{\ln \left(\left\langle R_{n}^{2}\right\rangle /\left\langle R_{m}^{2}\right\rangle\right)}{\ln (n / m)},
$$

we obtain estimates for $v$ that for short lengths start around 0.5 (the free random walk value), but for higher $n$ and $m$ these estimates are around 0.7. Extrapolations yield indeed values $v \simeq 0.75 \pm 0.02$, so that we may conclude that there is no doubt left about the fact that $k$ tolerant walks for finite $k$ belong to the SAW universality class and have the same fractal dimension.

On the same sample of 5-tolerant walks, we have also verified whether the number of $m$-multiple points $(m \leq 5)$ on a 5-tolerant walk grows linearly with the number $n$ of steps in the walk. This law seems indeed to hold, although again the linear behavior manifests itself only at higher $n$ values than in the 2-tolerant case. Our extrapolations for the 5-tolerant walks on the square lattice yield

$$
\left\langle\|W\|_{m}\right\rangle_{(|W|=n)} \simeq n A_{m},
$$

with

$$
\begin{aligned}
& A_{1}=0.2495 \pm 0.0005, \\
& A_{2}=0.1431 \pm 0.0005, \\
& A_{3}=0.0759 \pm 0.0001, \\
& A_{4}=0.0378 \pm 0.0001, \\
& A_{5}=0.0172 \pm 0.0001 .
\end{aligned}
$$

Obviously, we have that

$$
\sum_{m=1}^{5} m A_{m}=1
$$

[compare Eq. (3.15)]. It should be remarked that the decrease of the $A_{m}$ values with increasing $m$ is qualitatively similar to the behavior predicted by (3.15). An approximate quantitative similarity can be obtained, when we assume for $G(0,0)$ a value in the neighborhood of 2.0.

In order to stress the difference between the selfintersections and the mutual intersections, we have also investigated the sets of mutual intersection points of these 5-tolerant walks. Since they all start at the same origin, we neglected the first 10 steps of these walks, minimizing in this way the bias introduced by this common starting point. In analogy with (4.7), we define for two independent walks $W$ and $W^{\prime}$ the number of $m_{1}$-multiple points of $W$, which are at the same time $m_{2}$-multiple points of $W^{\prime}$ as

$\left\langle\left\|W_{m_{1}} \cap W_{m_{2}}^{\prime}\right\|\right\rangle_{\left(|\boldsymbol{W}|=\left|W^{\prime}\right|=n\right)}=n A\left(n ; m_{1}, m_{2}\right)$.

It is clear that

$$
\sum_{m_{2}=0}^{5} A\left(n ; m_{1}, m_{2}\right)=A_{m_{1}} \text {. }
$$

In contrast to $A_{m}$, however, these coefficients $\boldsymbol{A}\left(n ; m_{1}, m_{2}\right)$ do not tend to finite values when $n$ goes to infinity (except for $m_{1}=0$ or $m_{2}=0$ ), but they decrease to zero (see Table II). From a log-log analysis, one may deduce that for $n \rightarrow \infty$,

$$
A\left(n ; m_{1}, m_{2}\right) \sim n^{-\alpha} \quad\left(\text { if } m_{1} m_{2} \neq 0\right),
$$

where $a=0.5 \pm 0.1$. This estimate was obtained from $A(n ; 1,1)$; the other $m$ values produce larger uncertainties. This value of $a$ may simply be understood from the codimension additivity rule. Indeed, we remarked above

that

$$
\left\langle\left\|W_{m}\right\|\right\rangle_{(|W|=n)} \sim \xi^{D} \sim n,
$$

and thus, from (2.11),

$$
n A\left(n ; m_{1}, m_{2}\right) \sim \xi^{2 D-d} \sim n^{2-d / D},
$$

which means that

TABLE II. Some numerical estimates for $A\left(n ; m_{1}, m_{2}\right)$, the average density of common $m_{1}$ multiple and $m_{2}$-multiple points of two independent 5-tolerant walks of length $n$ on the square lattice.

\begin{tabular}{cccccc}
\hline \hline$n$ & 10 & 30 & 50 & 300 & 500 \\
\hline$A(n ; 1,0)$ & 0.40449 & 0.292547 & 0.265728 & 0.23488 & 0.23197 \\
$A(n ; 1,1)$ & 0.70776 & 0.038753 & 0.030692 & 0.014368 & 0.010436 \\
$A(n ; 1,2)$ & 0.02598 & 0.018617 & 0.015268 & 0.008264 & 0.006376 \\
$A(n ; 1,3)$ & 0.00789 & 0.008090 & 0.007466 & 0.004279 & 0.003504 \\
$A(n ; 1,4)$ & 0.00166 & 0.003113 & 0.003274 & 0.002078 & 0.001691 \\
$A(n ; 1,5)$ & 0.00021 & 0.001180 & 0.001256 & 0.000914 & 0.000699 \\
\hline \hline
\end{tabular}




$$
\alpha=\frac{d}{D}-1
$$

For $d=2$ and $D=\frac{4}{3}$, this gives $\alpha=\frac{1}{2}$.

\section{MUTUAL AND SELF-INTERSECTIONS AND FLORY APPROACH TO EXCLUDED-VOLUME PROBLEMS}

Flory approximations are a well-established tool in polymer physics and lattice statistics. Their success is often so remarkable and unexpected that naturally the problem arises of understanding the deep reasons for it. These reasons should somehow underlie the relatively simple standard derivation of Flory formulas.

One of the main results of the present paper is the clarification of the close connection between the Flory approach, and the statistical geometry of intersections of random fractals. In the authors' opinion, such connection is a first important step towards a credible justification for the success of the approach. What we are going to present below, amounts to an alternative new derivation of the results given by the Flory approximation: this derivation has also the considerable advantage of making the application of the method correct and successful, even in cases in which standard treatments run into serious ambiguities and difficulties. This is in particular the case for some recent attempts to treat $k$-tolerant lattice walks, ${ }^{4}$ or kinetic growth walks. ${ }^{18}$

The concept of self-intersections of a random fractal is at the basis of the Flory estimate of the free energy for a polymer in a good solvent. Let us consider the example of a linear polymer, which is well schematized by lattice random walks in $d$ dimensions, subject to the constraint of not visiting any point more than once (self-avoiding walks). The free energy at a given temperature and number of monomers (proportional to the number $N$ of steps in the representative lattice walk) is parametrized in terms of the end-to-end elongation $\xi$. The repulsive energy, due to the monomer-monomer repulsions is simply estimated as proportional to the average number of self-intersections that a free random walk, with the same elongation $\xi$, would possess. This number is thus $\sim N^{2} \xi^{-d}$, i.e., the number of monomers times an average monomer density $N \xi^{-d}$, since $\xi$ determines the spacial size of the chain. From this mean-field repulsive energy, one must subtract the elastic entropy $\sim \xi^{2} N^{-1}$, appropriate for a free random walk of elongation $\xi$. Extremalization of this free energy with respect to $\xi$ leads to the results

$$
N \sim \xi^{D} \quad \text { as } \mathrm{N} \rightarrow \infty
$$

with

$$
D=(2+d) / 3
$$

for $d \leq 4$ (and $D=2$ for $d>4$ ).

The derivation, sketched above, is the standard one for the result (5.2), whose spectacular agreement with the properties of the $d$-dimensional SAW is well known. Particularly surprising is the fact that, essentially on the basis of mean-field arguments, one obtains a remarkably accurate prediction of nontrivial scaling behavior.
It is crucial to notice that the Flory estimate of the repulsive energy as $\sim N^{2} \xi^{-d}$ is apparently inconsistent with the properties of self-intersections discussed in Sec. IV. We know, e.g. for $d>2$, that the number of multiple points of a random walk should be proportional to $N$. The repulsive energy $\sim N^{2} \xi^{-d}$ can be written as $\sim \xi^{2 D-d}$, so that, consistent with the law of codimension additivity, it should rather be interpreted as being proportional to the number of mutual intersections of two independent replicas of the random chain. The apparent contradiction between the above two aspects (mutual versus selfintersections) can be reconciled in the light of our new derivation.

In order to obtain the Flory free energy of a selfrepelling chain of $N$ monomers, let us first consider explicitly the case $d \geq 2$. The crucial ingredient of the new analysis is a coarse-graining of the description. We imagine we divide our chain into $N / l$ segments of $l$ steps, with $N \gg l \gg 1$. The free energy will then be equal to the sum of the free energies $F_{0}(l)$ of each separate segment, plus the extra contribution due to the interactions among the segments, and the entropy of their configuration. The first term is independent of the actual end-to-end elongation $\xi$ of the walk. In the spirit of the Flory approach, we treat the segments as free random walks of typical elongation $\sqrt{l}$. The segments can be conceived as steps in a coarsegrained walk. The mutual repulsive energy among segments is basically given by the average number of segment-segment encounters, $(N / l)^{2}(\xi / \sqrt{l})^{-d}$, multiplied with the average number of mutual intersections among two different segments, which come into contact. In this estimate, the end-to-end elongation has to be measured in terms of the typical segment length $\sqrt{l}$, in order to give the density of segments. Since each segment is itself a fractal with fractal dimension $2(d>2)$, we can estimate the number of intersections between two segments in contact as proportional to $(\sqrt{l})^{2+2-d}$, where we applied the law (2.11) of codimension additivity. The entropy of the segments can finally be given as that pertaining to a Gaussian chain of $N / l$ segments, and total elongation $\xi / \sqrt{l}$, i.e., $\sim(\xi / \sqrt{l})^{2} /(N / l)$. The total coarse-grained free energy can thus be written as

$$
\begin{aligned}
F= & \frac{N}{l} F_{0}(l)+a\left(\frac{N}{l}\right)^{2}\left[\frac{\xi}{\sqrt{l}}\right]^{-d}(\sqrt{l})^{4-d} \\
& +b\left[\frac{\xi}{\sqrt{l}}\right]^{2} /\left[\frac{N}{l}\right],
\end{aligned}
$$

where $a$ and $b$ are suitable dimensional factors, possibly embodying a weak dependence on $l$.

It is quite remarkable that the basic $\xi$ and $N$ dependences in Eq. (5.3) are the same as in the usual Flory free energy, since the parameter $l$ is essentially disappearing from the expression. As a consequence, the above derivation, whose advantages will be made more clear below, leads to the same SAW fractal dimension as the standard Flory argument. We learned, at this stage, that there is a nontrivial scaling-invariance property hidden in the usual form of the Flory free energy. This scaling invariance, highlighted here by a proper application of laws of the in- 
tersection geometry of random fractals, is analogous to the property of the free energy of, e.g., a magnetic system near the fixed point of a renormalization-group transformation. There is little doubt that the scaling-invariance principle expressed by Eq. (5.3) should be largely responsible for the accuracy of the Flory predictions.

In order to understand why the above argument also works for $d<2$, where the multiple points in a free random walk behave quite differently from the $d>2$ case [see Eq. (3.19)], it is convenient to investigate in a more detailed way the validity of (5.3). This will at the same time clarify the application of our approach to more controversial cases, like $k$-tolerant walks. Indeed, in supposing that the interaction between two segments of the walk is proportional to $(\sqrt{l})^{4-d}$, we actually omitted to discuss the precise way in which this proportionality constant builds up.

Suppose that we are dealing with two-body monomermonomer interactions (which is the natural assumption with self-avoiding walks). It is clear that contributions to the segment-segment repulsions arise, in general, from contacts of $m$-multiple points in one segment with $n$ multiple points in the other segment. Each such contact gives a contribution to the total segment-segment repulsion, roughly proportional to $n \mathrm{~m}$. Let us introduce a fractal density $C(l, m)$ for $m$-multiple points, such that the number of such points on a segment of length $l$ is

$$
\left\langle\|W\|_{m}\right\rangle_{l} \simeq C(l, m)(\sqrt{l})^{D_{m}},
$$

where $D_{m}$ is the fractal dimension of these multiple points [see Eq. (3.19)]. Since

$$
\sum_{m}\left\langle\|W\|_{m}\right\rangle_{l} m=l
$$

the densities $C(l, m)$ must satisfy

$$
\sum_{m} m C(l, m) \simeq l^{1-D_{m} / 2}
$$

The repulsive energy of a segment-segment contact can then be estimated as follows, using the codimension additivity rule (2.10),

$$
\sum_{m} \sum_{n} m n C(l, m) C(l, n)(\sqrt{l})^{2 D_{m}-d} \simeq l^{2-d / 2} .
$$

For $d>2$, the $C(l, m)$ are exponentially decreasing with $m$ [see (3.15)]; for $d<2$, however, the $C(l, m)$ become independent of $m$ in the $l \rightarrow \infty$ limit, and the intrinsic cutoff $m<l$ has to assure the convergence of the summation (5.6). In both cases, we recover a factor $(\sqrt{l})^{4-d}$ for the segment-segment repulsion, and (5.3) is generally valid.

The above derivation again shows how the important facts concerning intersections of free random walks, summarized in Sec. IV, are crucial for a correct discussion of the coarse-grained Flory free energy.

The usefulness of this scheme of reasoning can best be exemplified by considering the problem of $k$-tolerant walks on the lattice. These are defined as walks with the constraint that each lattice site may be visited at most $k$ times $(k=1,2, \ldots) . k=1$, of course, gives the SAW case. For $k>1$, the model represents a way of schema- tizing an excluded-volume effect different from the SAW. A question debated in the recent literature has been whether the asymptotic properties could be different for different $k$ 's. While there seems to be no doubt that the answer to this question is negative, on the basis of numerical and renormalization-group evidence ${ }^{17}$ it remains an open question how one can understand this property within a Flory strategy.

Recently, a proposal ${ }^{4}$ was made for a Flory free energy, in which the repulsive term was estimated as being proportional to $N\left(N / \xi^{d}\right)^{k}$. This choice, which is very natural within the standard deviation of Flory formulas, is, however, implying a $k$-dependent $D$, different from (5.2), and in open contradiction with the numerical and series results.

A way out of this contradiction can naturally be obtained in the framework of our coarse-grained Flory approach. We know that, even if the excluded-volume effects are felt microscopically only at a $(k+1)$-body level, the fractal dimensions of multiple self-intersection points, contributing to expressions like (5.4), is the same for all multiplicities. Thus, whatever the value of $k$, up to trivial $k$-dependent rescaling factors, the total repulsive energy between two segments is still proportional to $l^{2-d / 2}$, as in the SAW case and the usual result (5.2) applies.

In this way we realize that the coarse graining, at the basis of the present derivation of the Flory free energy, is most adequate for putting in evidence the universality properties of different microscopic excluded-volume mechanisms.

Along lines very similar to those presented here, it has also been shown ${ }^{19}$ that other, dynamic, walk problems, like kinetic growth walks, fall into the same universality class of static self-avoiding walks. This circumstance, which is supported by recent numerical estimates of $D$, was previously excluded, also on the basis of an application of the Flory approach in a standard form ${ }^{20}$ which suffered from much the same inconsistencies as that of Ref. 4.

\section{THE SPECTRAL DIMENSION OF RESTRICTED RANDOM WALKS}

Let us consider the diffusion of a particle on a random walk, whose fractal dimension is $D$. In general, $D$ may be different from $\bar{d}=v^{-1}$, where $\bar{d}$ is defined through

$$
\langle|W|\rangle \sim \xi^{\bar{d}}
$$

[compare with Eq. (2.6)], or equivalently

$$
\langle R\rangle_{|W|=n} \sim n^{v},
$$

where $R$ is the end-to-end distance of a walk.

The sites of a walk have a natural ordering by an index $j$, referring to the consecutive steps in the walk (intersection points then have multiple indices). If we suppose that a particle can diffuse only along this natural path, without profiting from the self-intersections, i.e.. with equal probabilities for going from site $j$ towards $j-1$ or $j+1$, it then moves a distance $\Delta j=n \sim \sqrt{t}$ after $t$ diffusion steps (we call $t$ the "time"). This means that the particle moves over a Euclidean distance $R \sim n^{1 / \bar{d}} \sim t^{1 / d_{w}}$, where $d_{w}=2 \bar{d}$ is 
the diffusion exponent. This trivial situation will be called "normal diffusion," in the sense that it occurs on a structure with the topology of a line.

This result is more transparent in terms of the vibrational problem. Indeed, after substituting the first time derivative by a second derivative, the diffusion equations map into the equations of motion for masses, where the hopping rates become elastic constants. ${ }^{21,5}$ The behavior of the density of vibrational states $\rho(\omega)$ at low frequencies $\omega$ defines the spectral dimension $\widetilde{d}$ (Refs. 22 and 5) through

$$
\rho(\omega) \sim \omega^{\bar{d}-1} \text {. }
$$

This satisfies the scaling relation

$$
\widetilde{d}=2 \bar{d} / d_{w} .
$$

The fact that, in the "normal" case $d_{w}=2 \bar{d}$ implies the obvious result $\widetilde{d}=1$.

More interesting, and less trivial, is the case of a particle that can profit from the self-intersections of the random walk by freely diffusing along all bonds visited by this walk. For $k$-tolerant walks $(k>1)$, the analysis of Sec. V, and the fact that these walks belong to the SAW universality class, ${ }^{17}$ indicates that loops are irrelevant to the asymptotic critical behavior. A natural question is whether these loops are also irrelevant for the dynamical properties of these fractals. The extensive numerical studies, which will follow in Sec. VI A, confirm that this is indeed the case.

In Sec. VI B we also analyze the problem on a SAW of a particle, which is allowed to jump to all neighboring sites visited by the walk, even if these sites are not directly linked by single walk steps to the actual site. Coarsegraining arguments would predict that this problem should have the same spectral properties as the diffusion on a $k$-tolerant walk.

\section{A. Spectral dimension of 2-tolerant walks}

We constructed on the square lattice a large sample (200000) of 2-tolerant walks of 200 steps, and we simulated 50 independent diffusion processes of 50 steps on each of these walks. The prescription for the diffusion was such that on a twice-visited point the particle got a probability of $25 \%$ for going into the direction of each of the four paths joining this point, even if two of these paths were identical. (We believe, however, that our results do not depend on such details of the diffusion mechanism.) On the constructed diffusion, we calculated the average square distances $\left\langle R^{2}(t)\right\rangle$ after $t$ steps, where in averaging we took into account the relative weights of the 2 -tolerant walks. ${ }^{16}$

The standard method of analysis, if applied to this series $\left\langle R^{2}(t)\right\rangle$ for $1 \leq t \leq 50$, show in a consistent way that asymptotically

$$
\left\langle R^{2}(t)\right\rangle \sim t^{0.7 \pm 0.1} .
$$

As explained in the beginning of this section, on a SAW we must have

$$
\left\langle R^{2}(t)\right\rangle \sim t^{1 / \bar{d}}
$$

and in $d=2$, this means $t^{3 / 4}$. Within the obtained accuracy we see no difference between the asymptotic behavior of the diffusion on a SAW and on a 2-tolerant walk. Relation (6.4) then leads to a spectral dimension $\widetilde{d}=1$, also for these 2-tolerant walks.

\section{B. Spectral dimension for SAW with extra hopping}

A SAW may return to the neighborhood of a point that was already visited. If we look upon such a near return in a coarse-grained way, or if we would apply some kind of cluster-renormalization procedure, this must resemble a self-intersection of the walk. This argument, which is at the basis of the intuitive proof for the fact that $k$-tolerant walks and SAW's belong to the same universality class, would also predict that the asymptotic properties of the diffusion on a SAW should be rather insensitive to small relaxations in the diffusion restrictions. We can, e.g., give equal probabilities to jump to all nearest-neighbor sites visited by the SAW, whether they are directly linked to the present site by a single walk step or not. We have tested this model, in which hopping over such bridges is allowed, by generating 50 diffusions of 50 steps on a large sample $(100000)$ of SAW's of length 200 on the square lattice. Our methods of series analysis indicate here

$$
\left\langle R^{2}(t)\right\rangle \sim t^{0.77 \pm 0.05}
$$

again compatible with (6.6), $d_{w}=2 \bar{d}$ and $\widetilde{d}=1$.

We have also tried to generalize this hopping possibility by allowing jumps over larger distances with decreasing probabilities. In this way we hoped to recover the nonuniversal spectral dimension that was found analytically in deterministic fractals with long-range interactions. ${ }^{23}$ At every step of the diffusion we scanned up to five concentric shells of neighboring sites, and allowed jumps to all the sites from these shells, that belonged to the original SAW, while the jump probability depended on the number of the shell. We were, however, unable to discover in this way a deviation from the previous result $\widetilde{d}=1$. It is not clear whether this is due to the limited nature of our simulations or to a true absence of nonuniversality in such long-range jump models.

\section{SPECTRAL DIMENSION OF FREE RANDOM WALKS}

The diffusion of a particle on a free random walk, where multiple intersections can be exploited to move between different parts of the walk, is more controversial than the problems treated above. The dynamical properties connected to free walks seem to present some surprising aspects, in contrast to their well-understood static properties.

As an example, let us consider the two-dimensional free random walks. The results of Sec. III show that, apart from logarithmic corrections, the number of multiple intersections is of the same order as the number of steps, and these intersection points are distributed uniformly over the volume of the walk. Since these walks are space filling $(\bar{d}=2)$, one is tempted to conclude that also $d_{w}=2=\widetilde{d}{ }^{9}$ There is, however, some evidence that things 
are not so simple. ${ }^{8}$ Although the probability that a infinite random walk ( $\mathrm{RW}$ ) in $d=2$ reaches some arbitrarily chosen lattice site is equal to unity, nevertheless each individual walk of finite, but eventually very large, size does contain a large number of vacancies and holes, both in occupied sites and in visited bonds. If the structure of these vacancies acquires some self-similarity over a wide range of length scales, as is suggested by inspection of some samples of these RW's, this may result in an anomalous spectral behavior. Such a behavior would be manifested in a range of $t$-values with an upper boundary $t_{\max } \sim R^{d_{w}} \sim N^{d_{w} / 2}$ (where $N$ is the number of steps in the RW). The asymptotic spectral behavior has to be understood for large $t$ values, but with $t<t_{\max }$. We have undertaken an accurate numerical analysis of this problem, and we tried to suggest a possible heuristic understanding of the results. ${ }^{24}$

\section{A. Numerical analysis}

We have generated a sample of about 5000 free random walks of 20000 steps on a square lattice of $512 \times 512$ sites with periodic boundary conditions. On each one of these random walks we generated 100 diffusion processes of 100 steps each. The rules for this diffusion were as follows. After every step, we counted the number of outgoing bonds visited by the original walk (in either direction), and the next step was taken with equal probabilities for each of these visited bonds. This means the following.

(1) the step probability is independent of the number of times such a bond has been visited,

(2) jumps to neighboring visited sites are not allowed if the connecting bond was not visited by the random walk.

We then calculated $\left\langle R_{n}^{2}\right\rangle$, the average square distance after $n$ steps for all these diffusion paths.

Since the standard methods of series analysis proved to yield rather varying results, and since logarithmic corrections are known to be present for the free random walk in two dimensions [see Eq. (3.14)], we suspected logarithmic corrections also in our results. In the spirit of the method used, e.g., for two-dimensional percolation, ${ }^{25}$ we investigated the $(N, M)$-Padé approximants to

$$
\left[(1-x) \frac{d}{d x} \ln \sum_{n} x^{n}\left\langle R_{n}^{2}\right\rangle(\ln n)^{-\alpha}\right]_{x=1}
$$

and we found that these Padé tables showed a very small variance only for a small range of $\alpha$ values (see Table III). From these tables, and from other methods of analysis applied to the series built with the coefficients $\left\langle R_{n}^{2}\right\rangle(\ln n)^{-\alpha}$, we conclude that

$$
\left\langle R_{n}^{2}\right\rangle \sim n^{\beta}(\ln n)^{\alpha},
$$

where $\alpha \simeq 0.70 \pm 0.05$ and $\beta \simeq 0.65 \pm 0.02$. These numbers suggest that, apart from a logarithmic correction, the dimension of the diffusion walk is given by $d_{w}=3$, and consequently its spectral dimension by $\widetilde{d}=\frac{4}{3}$. From the above numbers, it seems indeed reasonable to conjecture that $\alpha=\beta=\frac{2}{3}$.

\section{B. Heuristic argument}

It is possible to present a heuristic explanation for the results obtained above, on the basis of the relation between the spectral dimension $\widetilde{d}$ and the resistivity exponent $\zeta$, defined through

$$
\Omega(r) \sim r^{\zeta}
$$

where $\Omega(r)$ is the electrical resistance between two points at distance $r$ on the fractal, if the bonds of the fractal structure are replaced by identical resistances (each bond gets one unit resistance, independent of the number of times it was visited by the original random walk). It is known ${ }^{26}$ that

$$
\zeta=d_{w}-\bar{d}
$$

as a consequence of the Einstein relation for the conductivity $\sigma$ as a function of the density $n$ and the diffusion coefficient $D$,

$$
\sigma=\frac{e^{2}}{k_{B} T} n D
$$

\begin{tabular}{|c|c|c|}
\hline$\alpha$ & $\beta$ & $\sigma$ \\
\hline 0 & 0.7209 & 0.615056 \\
\hline 0.10 & 0.6155 & 1.081591 \\
\hline 0.20 & 0.7671 & 0.076961 \\
\hline 0.30 & 0.7382 & 0.022431 \\
\hline 0.40 & 0.7133 & 0.004381 \\
\hline 0.50 & 0.7059 & 0.084829 \\
\hline 0.52 & 0.6879 & 0.005235 \\
\hline 0.54 & 0.6833 & 0.003968 \\
\hline 0.56 & 0.6790 & 0.004340 \\
\hline 0.58 & 0.6750 & 0.005416 \\
\hline 0.60 & 0.6710 & 0.006505 \\
\hline 0.62 & 0.6666 & 0.004471 \\
\hline 0.64 & 0.6621 & 0.001410 \\
\hline 0.66 & 0.6579 & 0.000327 \\
\hline 0.68 & 0.6538 & 0.000106 \\
\hline 0.70 & 0.6498 & 0.000125 \\
\hline 0.72 & 0.6458 & 0.000144 \\
\hline 0.74 & 0.6415 & 0.001838 \\
\hline 0.76 & 0.6379 & 0.000232 \\
\hline 0.78 & 0.6335 & 0.003370 \\
\hline 0.80 & 0.6301 & 0.000941 \\
\hline 0.82 & 0.6267 & 0.002351 \\
\hline 0.84 & 0.6227 & 0.003835 \\
\hline 0.86 & 0.6193 & 0.005869 \\
\hline 0.88 & 0.6161 & 0.010794 \\
\hline 0.90 & 0.6151 & 0.030352 \\
\hline 1.00 & 0.5920 & 0.026387 \\
\hline 1.10 & 0.5583 & 0.027118 \\
\hline 1.20 & 0.5465 & 0.009301 \\
\hline 1.30 & 0.5178 & 0.009722 \\
\hline 1.40 & 0.4897 & 0.008636 \\
\hline
\end{tabular}

Indeed, since

TABLE III. Estimates for the exponent $\beta$ from Eq. (7.2), and their variance $\sigma$ over a central section of 50 elements of the Padé table to (7.1), as a function of the exponent $\alpha$. 


$$
D=\frac{d}{d t}\left\langle r^{2}(t)\right\rangle
$$

and

$$
\left\langle r^{2}(t)\right\rangle \sim t^{2 / d_{w}}
$$

we know that the diffusion constant at a distance $r$ on the fractal will scale as

$$
D \sim t^{2 / d_{w}-1} \sim r^{2-d_{w}} .
$$

The density $n$ obviously behaves like

$$
n \sim r^{\bar{d}-d}
$$

and thus

$$
\sigma \sim r^{\bar{d}-d_{w}+2-d}
$$

On the other hand, the resistance $\Omega(r)$ between two points at a distance $r$ is related to the conductivity through

$$
\Omega(r) \sim \frac{r}{\sigma} / r^{d-1} \sim r^{d_{w}-\bar{d}},
$$

which yields (7.4).

In order to have an independent estimate of $\Omega(r)$, we use the following picture. If the fractal is sufficiently dense, which is certainly the case for random walks in $d=2$, we can imagine that between two points $A$ and $B$ at a distance $r$ on the fractal, there exist several independent paths that can carry electrical current, when a voltage is applied. We may delete from the fractal all parts that do not transfer this current, such as dead-end branches and links between equal-potential points. We may then suppose that the remaining structure

(1) still has a number of bonds proportional to $r^{\bar{d}}$ (also in percolation, the collection of these paths is having almost the fractal dimension of the whole cluster),

(2) consists of a number of independent paths, which look very much like self-avoiding walks.

Each path is constructed of a number of bonds given by

$$
n_{b} \sim r^{d_{\mathrm{SAW}}}
$$

where $d_{\text {SAW }}$ is the fractal dimension of a SAW on the free random walk structure. The number of such paths should then be given by

$$
N \sim r^{\bar{d}} / n_{b} \sim r^{\bar{d}-d_{\mathrm{SAW}}} .
$$

The total resistance of this network is then

$$
\Omega(r) \sim \frac{n_{b}}{N} \sim r^{2 d_{\mathrm{SAW}}-\bar{d}}
$$

In combination with (7.4), this means that

$$
2 d_{\mathrm{SAW}}=d_{w} \text {. }
$$

Since the paths joining the two points are self-avoiding walks on the fractal, we can try to construct a Flory argument for obtaining $d_{\mathrm{SAW}}$. In this case we are not going to express in detail the coarse-graining procedure of Sec. V, which is, however, fully consistent with the results given below. It is rather natural to accept that the repulsive energy of a polymer on a fractal should in general be estimated as $\sim N^{2} / \xi^{\bar{d}}$, where $N$ is the number of steps, $\xi$ the elongation of the SAW, and $\bar{d}$ the fractal dimension of the random set, on which the SAW is constructed. In our case $\bar{d}=2$. Assuming such interaction energy implies a natural generalization of the codimension additivity rule, used for the segment-segment intersections, to a case in which the embedding space is itself a fractal. What is less obvious, in the case of a SAW on a fractal, is the choice of the entropic term. In the recent literature, some proposals have been made ${ }^{27,28}$ but there seems to be no fully satisfactory prescription valid for all cases. The crucial question is whether one can still assume a purely Gaussian distribution for the end-to-end elongation of a random walk on the random fractal or not. Arguments were recently produced in favor of stretched Gaussian distributions. The Flory results obtained by such assumptions, however, are often not very satisfactory. ${ }^{28}$ On the contrary, we recently obtained evidence that, at least in the case of fractals with $\bar{d}$ not too different from $d$, like suitable Sierpinsky gaskets in $d=2$ and 3 , or the backbone of the incipient infinite cluster of percolation at threshold in $d=2$, an assumption of a Gaussian distribution gives remarkably good results within the Flory context. On the basis of this assumption, which turns out to be most plausible for the case of the random walk in two dimensions, one can estimate the entropic term as $\sim \xi^{2} / N^{2 / d_{w}}$. The denominator in this expression is the mean square displacement of the random walk on the fractal, and $d_{w}$ is related ${ }^{5}$ to the usual dimensions by the relation $d_{w}=2 \bar{d} / \widetilde{d}$. The Flory free energy

$$
F=a \frac{N^{2}}{\xi^{\bar{d}}}+b \frac{\xi^{2}}{N^{2 \tilde{d} / \bar{d}}}
$$

leads to

$$
d_{\mathrm{SAW}}=\frac{2+\bar{d}}{2+\bar{d} / \bar{d}}=\frac{2+\bar{d}}{2\left(1+d_{w}^{-1}\right)}
$$

While we are conscious that a formula of this kind can hardly give satisfactory results for arbitrary ranges of $\bar{d}$ and $\bar{d}$, we know that its application to Sierpinsky gaskets in $d=2$, e.g., gives agreements with exact results for $d_{\text {SAW }}$ within less than $1 \%$. The same occurs for the backbone of percolation clusters in $d=2$.

Coming back now to the previously obtained equation (7.15), this produces in combination with our Flory expression for $d_{\text {SAW }}$ a self-consistency condition on $d_{w}$. Application of Eq. (7.17) imposes

$$
\frac{d_{w}}{2}=d_{\mathrm{SAW}}=\frac{2+\bar{d}}{2\left(1+d_{w}^{-1}\right)}
$$

or

$$
d_{w}+1=2+\bar{d},
$$

from which follows $\zeta=1$. If, furthermore, $\bar{d}=2$, as is the case for free random walks, we then immediately obtain $d_{w}=3, \widetilde{d}=\frac{4}{3}$, and this agrees with our numerical result 
described above.

A value $\zeta=1$ has been obtained independently by direct numerical investigation of the resistance $\Omega(r)$ (Ref. 8) in a model where a resistivity $q^{-1}$ was attributed to each bond that was visited $q$ times by the random walk. In the model used in our numerical investigation and in the heuristic argument we should give equal resistances to all visited bonds. It turns out that the results are rather insensitive to such details.

\section{CONCLUSIONS}

In this paper, we have treated several fundamental properties of free and restricted random-walk fractals. We pointed out the important difference between the fractal dimensions of the set of self-intersection points of such fractals and the set of mutual intersections of two independent fractals. Our statements were confirmed by numerical simulations.

We have resolved an apparent controversy in the interpretation of the standard Flory approximation, where the repulsive-energy term seems not to be proportional to the number of self-intersections of the free walk. By a coarse-graining argument we argued that the standard picture is consistent with repulsions arising from mutual intersections between different independent segments of the same walk.

The universality of the class of self-avoiding and $k$ tolerant walks has been extended to their dynamical properties, through numerical investigations of the diffusion problem on such fractals. The same dynamical problem was finally studied on the free random-walk fractal structure in $d=2$, where we were able to give strong evidence for the conjecture that $d_{w}=3$ and $\widetilde{d}=\frac{4}{3}$, up to logarithmic corrections, which may confuse a too-primitive analysis. A heuristic argument for such dynamical behavior was presented, based on an extension of the Flory approach to self-avoiding walks on fractals, and on an intuitive picture for the resistivity in such disordered systems.

\section{APPENDIX: THE CORRELATION FUNCTIONS OF THE RANDOM WALK}

In this appendix we want to derive in more detail some correlation functions introduced in Secs. II and III. We define the following matrices (for $\mathbf{x}$ and $\mathbf{y}$ arbitrary sites on the lattice):

$$
K_{\mathbf{x}, \mathbf{y}}=\left\{\begin{array}{l}
K \text { if }|\mathbf{x}-\mathbf{y}|=1 \text { (nearest neighbors) } \\
0 \text { otherwise }
\end{array}\right.
$$

and

$$
\mathcal{I}_{\mathbf{x}, \mathbf{y}}=j_{\mathbf{x}} \delta_{\mathbf{x}, \mathbf{y}}
$$

It is rather straightforward that the Green function defined in (2.1) may be written as

$$
G(\mathbf{x}, \mathbf{y})=[1-\mathcal{K}]_{\mathbf{x}, \mathbf{y}}^{-1}
$$

and, more generally,

$$
\left[\mathcal{I}^{-1}-\mathcal{K}\right]_{\mathbf{x}, \mathbf{y}}^{-1} \sum_{\substack{W \\ \partial W=\{\mathbf{x}, \mathbf{y}\}}} K^{|W|} \prod_{\mathbf{z} \in W} j_{z}^{n_{z}},
$$

where $n_{z}$ is the number of times that the free walk visits the site $\mathbf{z}$. The correlation function $P_{m}(\mathbf{x})$, defined in (2.12), can then be written as

$$
\begin{aligned}
P_{m}(\mathbf{x})= & {\left[\sum_{\mathbf{y}} G(\mathbf{0}, \mathbf{y})\right]^{-1} } \\
& \times\left.\frac{1}{m !} \frac{\partial^{m}}{\partial j_{x}^{m}} \sum_{\mathbf{y}}\left[\mathcal{J}^{-1}-\mathcal{K}\right]_{0, \mathbf{y}}^{-1}\right|_{j_{z}=1-\delta_{x, z}} .
\end{aligned}
$$

In order to deduce some practical results from these equations, we introduce an $n$-component vector field $\phi_{\mathrm{x}}$ at every site $\mathbf{x}$, coupled to external fields $\mathbf{h}_{\mathbf{x}}$. Using the Hamiltonian

$$
\begin{aligned}
\mathcal{H}\{\boldsymbol{\phi}, \mathbf{h}\} \equiv & \frac{1}{2} \sum_{\mathbf{x}, \mathbf{y}} \sum_{\alpha=1}^{n} \varphi_{\mathbf{x} \alpha}\left[\mathcal{J}^{-1}-\mathcal{K}\right]_{\mathbf{x}, \mathbf{y}} \varphi_{\mathbf{y} \alpha}-\sum_{\mathbf{x}} \sum_{\alpha=1}^{n} \varphi_{\mathbf{x} \alpha} h_{\mathbf{x} \alpha} \\
= & -\frac{1}{2} \sum_{\mathbf{x}}\left(1-j_{\mathrm{x}}^{-1}\right) \varphi_{\mathbf{x}}^{2}+\frac{1}{2} \sum_{\mathbf{x}, \mathbf{y}}[1-\mathcal{K}]_{\mathrm{x}, \mathrm{y}} \boldsymbol{\varphi}_{\mathbf{x}} \cdot \varphi_{\mathbf{y}} \\
& -\sum_{\mathbf{x}} \varphi_{\mathbf{x}} \cdot h_{\mathbf{x}}
\end{aligned}
$$

one can easily prove the equation

$\left[\mathcal{I}^{-1}-\mathcal{K}\right]_{\mathbf{x}, \mathbf{y}}^{-1}=\left.\lim _{n \rightarrow 0} \frac{\partial^{2}}{\partial h_{x 1} \partial h_{y 1}} \int \mathcal{D} \boldsymbol{\varphi} \exp (-\mathscr{H}\{\boldsymbol{\varphi}, \mathbf{h}\})\right|_{\mathbf{h}_{z}=0}$,

where $D \varphi=\Pi_{\mathrm{z}} d^{n} \varphi_{\mathrm{z}}$.

We choose $j_{\mathbf{z}}=1$ for $\mathbf{z} \neq \mathbf{x}$ and $j_{\mathbf{x}}=j$, and we use the identity

$$
\begin{aligned}
\exp & {\left[\frac{1}{2} \boldsymbol{\varphi}_{\mathbf{x}}^{2}\left(1-\frac{1}{j}\right)\right] } \\
& =\left(\frac{j}{2 \pi(1-j)}\right)^{n / 2} \int d^{n} \boldsymbol{\alpha} \exp \left(-\frac{j}{1-j} \frac{\alpha^{2}}{2}+i \alpha \cdot \boldsymbol{\varphi}_{\mathbf{x}}\right),
\end{aligned}
$$

in order to write for this set $\left\{j_{z}\right\}$,

$$
\begin{aligned}
\int \mathcal{D} \boldsymbol{e} \exp (-\mathcal{H}\{\boldsymbol{\varphi}, \mathbf{h}\}) \\
=C^{n} \int d^{n} \boldsymbol{\alpha} \exp \left(\frac{1}{2} \sum_{\mathbf{y}, \mathbf{z}} \mathbf{b}_{\mathbf{y}} \cdot \mathbf{b}_{\mathbf{z}} G(\mathbf{y}, \mathbf{z})-\frac{\alpha^{2}}{2} \frac{j}{1-j}\right),
\end{aligned}
$$

where

$$
\mathbf{b}_{\mathrm{z}}=\mathbf{h}_{\mathrm{z}}+i \boldsymbol{\alpha} \delta_{\mathrm{z}, \mathbf{x}}
$$

and $C$ is a constant independent of the fields h. From this equation, we easily obtain 


$$
\begin{aligned}
& \left.\frac{\partial^{2}}{\partial h_{01} \partial h_{\mathrm{y} 1}} \int \mathcal{D} \boldsymbol{\varphi} \exp (-\mathcal{H})\right|_{\mathbf{h}=0} \\
& \left.=C^{n} \int d^{n} \boldsymbol{\alpha} \exp \left[-\frac{1}{2} \boldsymbol{\alpha}^{2} \mid G(\mathbf{x}, \mathbf{x})+\frac{j}{1-j}\right]\right] \\
& \quad \times\left[G(\mathbf{0}, \mathbf{y})-\alpha_{1}^{2} G(\mathbf{0}, \mathbf{x}) G(\mathbf{y}, \mathbf{x})\right],
\end{aligned}
$$

and taking the $n \rightarrow 0$ limit,

$$
\left[\mathcal{J}^{-1}-\mathcal{K}\right]_{0, \mathbf{y}}^{-1}=G(\mathbf{0 , y})-\frac{G(0, \mathbf{x}) G(\mathbf{y}, \mathbf{x})}{G(0,0)+j /(1-j)} .
$$

Using this result in (A5) we obtain

$$
\begin{aligned}
P_{m}(\mathbf{x}) & =-\left.\frac{G(\mathbf{0}, \mathbf{x})}{m !} \frac{\partial^{m}}{\partial j^{m}}\left[G(\mathbf{0 , 0})+\frac{j}{1-j}\right]^{-1}\right|_{j=0} \\
& =\frac{G(\mathbf{0}, \mathbf{x})}{G(\mathbf{0}, \mathbf{0})^{2}}\left[\frac{G(\mathbf{0 , 0})-1}{G(\mathbf{0 , 0})}\right]^{m-1} .
\end{aligned}
$$

This is Eq. (3.12).

A tedious but straightforward calculation along the same lines leads to $G_{m}(\mathbf{x}, \mathbf{y})$, defined in (2.15). Let us demonstrate this, e.g., for $m=2$. We choose $j_{z}=1$ for $\mathbf{z} \neq \mathbf{x}$ or $\mathbf{y}$. We may then write

$$
\begin{aligned}
\boldsymbol{G}_{2}(\mathbf{x}, \mathbf{y}) & =\left.\sum_{\mathbf{u}, \mathbf{v}}\left[\frac{1}{2 !}\right]^{2} \frac{\partial^{2}}{\partial j_{x}^{2}} \frac{\partial^{2}}{\partial j_{y}^{2}}\left[\mathcal{J}^{-1}-\mathcal{K}\right]_{\mathbf{u}, \mathbf{v}}^{-1}\right|_{j_{z}=1-\delta_{z, x}-\delta_{z, y}} \\
& =\left.\frac{1}{4} \sum_{\mathbf{u}, \mathbf{v}} \frac{\partial^{2}}{\partial j_{x}^{2}} \frac{\partial^{2}}{\partial j_{y}^{2}} \lim _{n \rightarrow 0} \frac{\partial^{2}}{\partial h_{u 1} \partial h_{v 1}} C^{n} \int d^{n} \boldsymbol{\alpha} \int d^{n} \boldsymbol{\beta} \exp \left(\frac{1}{2} \mathbf{b} G \mathbf{b}-\frac{\boldsymbol{\alpha}^{2}}{2} \frac{j_{x}}{1-j_{x}}-\frac{\boldsymbol{\beta}^{2}}{2} \frac{j_{y}}{1-j_{y}}\right)\right|_{\mathbf{h}=\mathbf{0} ; j_{x}=j_{y}=0},
\end{aligned}
$$

where $\mathrm{b}_{\mathrm{z}}=\mathbf{h}_{\mathrm{z}}+i\left(\boldsymbol{\alpha} \delta_{\mathrm{x}, \mathrm{z}}+\boldsymbol{\beta} \delta_{\mathrm{y}, \mathrm{z}}\right)$. From Eq. (A14) one may deduce that

$$
G_{2}(\mathbf{x}, \mathbf{y})=\frac{1}{2}\left(\sum_{\mathbf{z}} G(\mathbf{0}, \mathbf{z})\right)^{2}\left(\frac{\partial}{\partial G(\mathbf{0}, \mathbf{0})}+\frac{\partial}{\partial G(\mathbf{x}, \mathbf{y})}\right) \frac{G^{4}(\mathbf{x}, \mathbf{y})+2 G^{2}(\mathbf{x}, \mathbf{y})\left[G(\mathbf{0}, \mathbf{0})+G^{2}(\mathbf{0}, \mathbf{0})-G^{2}(\mathbf{x}, \mathbf{y})\right]^{2}}{\left[G^{2}(\mathbf{0}, \mathbf{0})-G^{2}(\mathbf{x}, \mathbf{y})\right]^{4}}
$$

Since $G(\mathbf{x}, \mathbf{y}) \rightarrow 0$ in the $|\mathbf{x}-\mathbf{y}| \rightarrow \infty$ limit, it follows immediately from (A15) that

$$
\begin{aligned}
G_{2}(\mathbf{x}, \mathbf{y})= & 2\left(\sum_{\mathbf{z}} G(\mathbf{0}, \mathbf{z})\right)^{2} \frac{[G(0,0)-1]^{2}}{G^{6}(0,0)} G(\mathbf{x}, \mathbf{y}) \\
& +O\left(G^{2}(\mathbf{x}, \mathbf{y})\right) \text { as }|\mathbf{x}-\mathbf{y}| \rightarrow \infty
\end{aligned}
$$

The formalism presented above allows also to calculate more general correlation functions for free random walks; it can, e.g., be applied to random walks, which visit a given set of points $\left\{\mathbf{x}_{i}\right\},(i=1,2, \ldots, N)$ a given number of times $n_{1}, n_{2}, \ldots, n_{N}$.
*Also at Sezione di Padova, Istituto Nazionale di Fisica Nucleare, I-3513 Padova, Italy.

${ }^{\dagger}$ Also at International School for Advanced Studies, I-34014 Trieste, Italy and Unità di Padova, Gruppo Nazionale di Struttura della Materia del Consiglio Nazionale delle Ricerche e Centro Interuniversitario di Struttura della Materia, I-35131 Padova, Italy.

${ }^{1}$ P. J. Flory, Principles of Polymer Chemistry (Cornell University Press, Ithaca, 1969).

${ }^{2}$ P. G. de Gennes, Scaling Concepts in Polymer Physics (Cornell University Press, Ithaca, 1979).

${ }^{3}$ E. W. Montroll and G. H. Weiss, J. Math. Phys. 6, 167 (1965).

${ }^{4}$ L. Turban, J. Phys. A 16, L643 (1983).

${ }^{5}$ S. Alexander and R. Orbach, J. Phys. (Paris) Lett. 43, L625 (1982).

${ }^{6}$ R. Rammal and T. Toulouse, J. Phys. (Paris) Lett. 44, L13 (1983).

${ }^{7}$ H. J. Stapleton, J. P. Allen, C. P. Flynn, D. G. Stinson, and S. R. Kurtz, Phys. Rev. Lett. 45, 1456 (1980).
${ }^{8}$ J. R. Banavar, A. B. Harris, and J. Koplik, Phys. Rev. Lett. 51, 1115 (1983).

${ }^{9}$ S. Havlin, G. H. Weiss, D. Ben-Avraham, and D. Moshovitz, J. Phys. A 17, L849 (1984).

${ }^{10}$ A. L. Stella, R. Dekeyser, and A. Maritan in Fractals in Physics, edited by L. Pietronero and E. Tosatti (North-Holland, Amsterdam, 1986).

${ }^{11}$ B. B. Mandelbrot, The Fractal Geometry of Nature (Freeman, San Francisco, 1982).

${ }^{12}$ S. J. Taylor, Proc. Cambridge Philos. Soc. 48, 31 (1953).

${ }^{13}$ K. J. Falconer, The Geometry of Fractal Sets (Cambridge University Press, Cambridge, 1985).

${ }^{14}$ P. Erdos and S. J. Taylor, Acta Math. Acad. Sci. Hung. 11, 137 (1960).

${ }^{15}$ See, e.g., K. Symanzik in Local Quantum Theory, Proceedings of the International School of Physics "Enrico Fermi," Course XLV, Varenna, 1969, edited by R. Jost (Academic, New York, 1969). For a more recent reference, see D. Bridges, J. Fröhlich, and T. Spencer, Commun. Math. Phys. 83, 123 
(1982).

${ }^{16}$ M. N. Rosenbluth and A. W. Rosenbluth, J. Chem. Phys. 23, 356 (1955).

${ }^{17}$ R. Dekeyser, A. Maritan, and A. L. Stella, Phys. Rev. B 31, 4659 (1985).

${ }^{18}$ I. Majid, J. Naeem, A. Coniglio, and H. E. Stanley, Phys. Rev. Lett. 52, 1257 (1984).

${ }^{19}$ A. L. Stella, Phys. Rev. Lett. 56, 2430 (1986).

${ }^{20}$ L. Pietronero, Phys. Rev. Lett. 55, 2025 (1985).

${ }^{21}$ S. Alexander, J. Bernasconi, W. R. Schneider, and R. Orbach, Rev. Mod. Phys. 53, 175 (1981).
${ }^{22}$ D. Dhar, J. Math. Phys. 18, 577 (1977).

${ }^{23}$ A. Maritan and A. L. Stella, Phys. Rev. B 34, 456 (1986).

${ }^{24} \mathrm{~A}$ preliminary report of this analysis was presented by $\mathbf{R}$. Dekeyser, A. Maritan, and A. Stella, Phys. Rev. Lett. 58, 1758 (1987).

${ }^{25}$ J. Adler and V. Privman, J. Phys. A 14, L463 (1981).

${ }^{26}$ Y. Gefen, A. Aharony, and S. Alexander, Phys. Rev. Lett. 50, 77 (1983).

${ }^{27}$ J. Vannimenus, J. Phys. (Paris) Lett. 45, L1071 (1984).

${ }^{28}$ R. Rammal, G. Toulouse, and J. Vannimenus, J. Phys. (Paris) 45, 389 (1984). 